\title{
Correction to "Isotopic characterization of aerosol organic carbon components over the eastern United States"
}

\author{
Andrew S. Wozniak, James E. Bauer, Rebecca M. Dickhut, Li Xu, and Ann P. McNichol \\ Received 12 July 2012; published 2 August 2012.
}

Citation: Wozniak, A. S., J. E. Bauer, R. M. Dickhut, L. Xu, and A. P. McNichol (2012), Correction to "Isotopic characterization of aerosol organic carbon components over the eastern United States", J. Geophys. Res., 117, D15399, doi:10.1029/2012JD018478.

[1] In the paper "Isotopic characterization of aerosol organic carbon components over the eastern United States" by A. S. Wozniak (Journal of Geophysical Research, 117,
D13303, doi:10.1029/2011JD017153, 2012), the Table 2 column headings "Millbrook" and "Harcum" were published incorrectly. The correct Table 2 appears here.

Table 2. Isotopic Signatures and Carbon Contents of Large-Volume Aerosol Samples Collected for Detailed Isotopic Analyses ${ }^{\mathrm{a}}$

\begin{tabular}{|c|c|c|c|c|c|c|c|c|c|c|}
\hline \multirow{2}{*}{$\begin{array}{l}\text { Aerosol OC } \\
\text { Component }\end{array}$} & \multirow[b]{2}{*}{ Parameter } & \multicolumn{4}{|c|}{ Millbrook } & \multicolumn{4}{|c|}{ Harcum } & \multirow[b]{2}{*}{ Overall Mean } \\
\hline & & March 7-10 & May $14-16$ & Aug 10-12 & Mean & Feb 19-21 & April 10-12 & Aug 6-8 & Mean & \\
\hline \multirow[t]{3}{*}{ TOC } & $\delta^{13} \mathrm{C}(\%)$ & -25.3 & -25.8 & -24.7 & -25.3 & -26.2 & -26.5 & -24.4 & -25.7 & -25.5 \\
\hline & $\Delta^{14} \mathrm{C}(\%)$ & -448 & -39 & 25 & -154 & -252 & -388 & -165 & -268 & -211 \\
\hline & $f_{O C}$ & 0.134 & 0.228 & 0.281 & 0.214 & 0.179 & 0.169 & 0.154 & 0.167 & 0.191 \\
\hline \multirow[t]{3}{*}{ WSOC } & $\delta^{13} \mathrm{C}(\%)$ & $\mathrm{nd}^{\mathrm{b}}$ & -25.1 & -24.4 & -24.7 & -26.1 & -24.8 & -25.2 & -25.4 & -25.1 \\
\hline & $\Delta^{14} \mathrm{C}(\%)$ & nd & -17 & 6 & -6 & 26 & 11 & 22 & 20 & 10 \\
\hline & $f_{W S O C}{ }^{c}$ & nd & 0.22 & 0.47 & 0.34 & 0.29 & 0.16 & 0.26 & 0.24 & 0.28 \\
\hline \multirow[t]{3}{*}{$\mathrm{WIOC}^{\mathrm{d}}$} & $\delta^{13} \mathrm{C}(\% 0)$ & nd & -26.0 & -25.1 & -25.6 & -26.7 & -26.8 & -24.2 & -25.9 & -25.8 \\
\hline & $\Delta^{14} \mathrm{C}(\%)$ & nd & -45 & 42 & -1 & -367 & -461 & -231 & -353 & -212 \\
\hline & $f_{W I O C}$ & nd & 0.78 & 0.53 & 0.66 & 0.71 & 0.84 & 0.74 & 0.76 & 0.72 \\
\hline \multirow[t]{3}{*}{$\mathrm{TSE}^{\mathrm{e}}$} & $\delta^{13} \mathrm{C}(\%)$ & -27.0 & nd & -26.2 & -26.6 & nd & -27.3 & -28.2 & -27.6 & -27.2 \\
\hline & $\Delta^{14} \mathrm{C}(\%)$ & -476 & nd & -90 & -283 & nd & -430 & -119 & -190 & -227 \\
\hline & $f_{T S E}$ & 0.67 & nd & 0.90 & 0.79 & nd & 0.43 & 0.74 & 0.59 & 0.69 \\
\hline \multirow[t]{3}{*}{ Aliphatic } & $\delta^{13} \mathrm{C}(\%)$ & -28.6 & -27.7 & $n d^{\mathrm{f}}$ & -28.2 & nd & -27.3 & -28.9 & -28.1 & -28.1 \\
\hline & $\Delta^{14} \mathrm{C}(\%)$ & -794 & -820 & -961 & -858 & nd & -834 & -858 & -846 & -853 \\
\hline & $f_{\text {aliphatic }}$ & 0.007 & 0.001 & 0.003 & 0.0039 & nd & 0.007 & 0.002 & 0.004 & 0.004 \\
\hline \multirow[t]{3}{*}{ Aromatic } & $\delta^{13} \mathrm{C}(\%)$ & -27.8 & -29.6 & -28.3 & -28.6 & -28.3 & -28.4 & -27.8 & -28.2 & -28.4 \\
\hline & $\Delta^{14} \mathrm{C}(\%)$ & -692 & -77 & -446 & -405 & -466 & -540 & -446 & -484 & -444 \\
\hline & $f_{\text {aromatic }}$ & 0.015 & 0.009 & 0.004 & 0.009 & 0.009 & 0.007 & 0.003 & 0.007 & 0.006 \\
\hline \multirow[t]{3}{*}{ Polar } & $\delta^{13} \mathrm{C}(\%)$ & -27.8 & -26.1 & -28.0 & -27.3 & -28.6 & nd & -26.2 & -27.4 & -27.3 \\
\hline & $\Delta^{14} \mathrm{C}(\%)$ & -750 & 24 & -93 & -273 & -168 & nd & -240 & -204 & -245 \\
\hline & $f_{\text {polar }}$ & 0.24 & 0.039 & 0.25 & 0.18 & 0.063 & nd & 0.066 & 0.065 & 0.13 \\
\hline
\end{tabular}

${ }^{\mathrm{a}} \mathrm{All}$ reported $\delta^{13} \mathrm{C}$ and $\Delta^{14} \mathrm{C}$ values were corrected for blank contributions following procedures outlined in the text.

b'nd' denotes samples for which values were not determined.

${ }^{c}$ Values represent the fraction of TOC accounted for by the parameter of interest $\left(f_{W S O C}, f_{W I O C}, f_{T S E}, f_{\text {aliphatic }}, f_{\text {aromatic }}, f_{\text {polar }}\right)$.

${ }^{\mathrm{d}}$ WIOC (water-insoluble organic carbon) values were calculated by mass balance using values for TOC and WSOC (WIOC $=$ TOC-WSOC): $X_{W I O C}=\frac{\left(X_{T O C} f_{T O C}-X_{W S O C} *_{W S O C}\right)}{f_{W I O C}}$, where $\mathrm{X}$ represents either $\delta^{13} \mathrm{C}$ or $\Delta^{14} \mathrm{C}$ for the component of interest (TOC, WSOC, WIOC).

${ }^{\mathrm{e}} \mathrm{TSE}=$ Total Solvent Extract.

${ }_{\mathrm{f}}^{\mathrm{f}}$ Sample was too small for measurement of both $\delta^{13} \mathrm{C}$ and $\Delta^{14} \mathrm{C}$. A value of $-25.0 \%$ was assumed for $\Delta^{14} \mathrm{C}$ fractionation corrections. 